\title{
Maximum Weighted Independent Set of Links under Physical Interference Model ${ }^{\star}$
}

\author{
Xiaohua Xu, Shaojie Tang, and Peng-Jun Wan \\ Illinois Institute of Technology, Chicago IL 60616, USA
}

\begin{abstract}
Interference-aware scheduling for wireless communications is crucial to improve the network throughput. In this paper, we study the problem of Maximum Weighted Independent Set of Links (MWISL) under the physical interference model in wireless networks. Given a set of communication links distributed in a two-dimensional Euclidean plane, assume each link is associated with a positive weight which represents the benefit of transmitting along the link, the objective is to seek an independent set of links subject to the physical interference constraints with maximum weighted sum. To the best of our knowledge, no algorithm for MWISL under physical interference model has been proposed. We focus on MWISL in the oblivious power assignment setting.
\end{abstract}

\section{Introduction}

Link scheduling in wireless networks plays a critical role for wireless networking performances, especially when the network has stringent quality of service restrictions. One challenge for link scheduling lies in interferences among concurrent transmissions. Unlike the wired networks, the signal interference casts significant effect on the fundamental limit on the data throughput that any scheduling protocols (centralized or distributed) can achieve. It is well-known that a number of scheduling problems (e.g., maximum throughput scheduling) become NP-hard to solve when considering wireless interference, while their counter-parts are solvable in polynomial time for wired networks. Thus, the scheduling protocols for wireless networks (even the benchmark performances obtained by centralized scheduling approaches) often rely on heuristics that approximately optimize the throughput.

We address a fundamental problem for scheduling in wireless communications: Maximum Weighted Independent Set of Links (MWISL): given an input set of links, a subset of links is an independent set of links iff they can transmit concurrently. Assume each link is associated with a positive weight (representing the benefit of transmitting along the link), the objective is to seek an independent set of links with maximum weighted sum under given interference model. We focus on MWISL under physical interference model, where a signal is received successfully if the Signal to Interferenceplus-Noise Ratio is above a threshold depending on hardware and coding method. In this practical model, the definition of a successful transmission accounts also interference generated by transmitters located far away. Thus, traditional methods in graphbased interference models (e.g., protocol interference model, RTS/CTS model et al.)

\footnotetext{
* This work was supported in part by NSF of USA under grant CNS-0831831.
} 
cannot be directly applied here. The MWISL problem has several variations: such as different power assignments and different weight distributions. Even for the special case where every link has unit weight, MWISL is proved to be NP-hard [6, 7].

Our main contribution is the algorithm design for MWISL problem under the oblivious power assignment setting where each link $l$ (or its corresponding sender) is assigned a transmission power $c \cdot\|l\|^{\beta}$, here $c$ is a constant and $0<\beta \leq \kappa$ is a constant. We can prove that our solution satisfies the interference constraints and achieves constant approximation ratio of the optimum when $\beta=\kappa$. Our main idea is like this: by using partition and shifting strategies, we find multiple sets of well-separated links and then select the one with the largest weight.

The rest of the paper is organized as follows. Section 2 formulates our problems. Section 3 presents our algorithm design for the MWISL problem. Section 4 outlines the related work. Finally, Section 5 concludes the paper.

\section{Network Model}

All the networking nodes $V$ lie in plane and have a maximum transmission power $P$. The Euclidean distance between any pair of nodes is denoted by $\|u v\|$. Let $r$ be the distance between a closest pair of nodes in $V$. The path-loss over a distance $l$ is $\eta l^{-\kappa}$, where $\kappa$ is path-loss exponent (a constant greater than 2 and 5 depending on the wireless environment), and $\eta$ is the reference loss factor. Since the path-loss factor over the distance $r$ is less than one, we have $\eta<r^{\kappa}$. In an oblivious power assignment, a node $u$ transmits to another node $v$ always at the power $c\|u v\|^{\beta}$ for some constants $c>0$ and $0<\beta \leq \kappa$. This assumption implicitly imposes an upper bound on the distance between a pair of nodes which directly communicate with each other: For $u$ to be able to directly communicate with $v$, we must have $c\|u v\|^{\beta} \leq P$ and hence $\|u v\| \leq(P / c)^{1 / \beta}$. Let $\xi$ be the noise power, and $\sigma$ be the signal to interference and noise ratio (SINR) threshold for successful reception. Then, in the absence of interference, the transmission by a node $u$ can be successfully received by another node $v$ if and only if $\frac{c\|u v\|^{\beta} \cdot \eta\|u v\|^{-\kappa}}{\xi} \geq \sigma$ which is equivalent to $\|u v\|^{\kappa-\beta} \leq \frac{c \eta}{\sigma \xi}$. Note that when $\|u v\|^{\kappa-\beta}=\frac{c \eta}{\sigma \xi}$, link $u v$ can only transmit alone since any other link will conflict with $u v$. Thus we can disregard these links in $A$ and assume that

$$
\|u v\|^{\kappa-\beta}<\frac{c \eta}{\sigma \xi}
$$

Therefore, the set $A$ of communication links consists of all pairs $(u, v)$ of distinct nodes satisfying that $\|u v\|^{\beta} \leq P / c$ and $\|u v\|^{\kappa-\beta}<\frac{c \eta}{\sigma \xi}$. Let $R$ be the maximum length of the links in $A$. Then,

$$
R^{\kappa}=R^{\beta} \cdot R^{\kappa-\beta}<\frac{P}{c} \cdot \frac{c \eta}{\sigma \xi}=\frac{P \eta}{\sigma \xi}<\frac{P}{\sigma \xi} r^{\kappa} .
$$

Hence,

$$
\frac{R}{r}<\left(\frac{P}{\sigma \xi}\right)^{1 / \kappa}
$$


A set $I$ of links in $A$ is said to be independent if and only if all links in $I$ can transmit successfully at the same time under the oblivious power assignment, i.e., the SINR of each link in $I$ is above $\sigma$. We denote by $\mathcal{I}$ the collection of independent sets of links in $A$. Given a link weight function $d \in \mathbb{R}_{+}^{A}$, the problem Maximum Weighted Independent Set of Links (MWISL) seeks a set $I \in \mathcal{I}$ with maximum total weight $d(I)=\sum_{a \in I} d(a)$.

\section{Algorithm Design}

In this section, we present our algorithm design for MWISL in the oblivious power assignment setting under physical interference model.

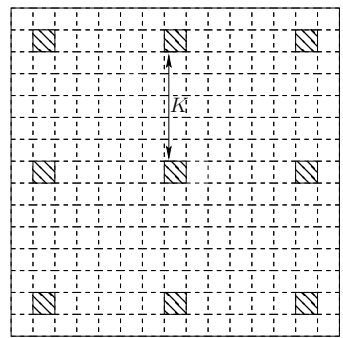

Fig. 1. Grid partition of the plane

We employ a grid partition of the plane (Fig. 1). Let $\ell=R / \sqrt{2}$. The vertical lines $x=i \cdot \ell$ for $i \in \mathbb{Z}$ and horizontal lines $y=j \cdot \ell$ for $j \in \mathbb{Z}$ partition the planes into half-open and half-closed grides of side $\ell$ (here $\mathbb{Z}$ represents the integer set):

$$
\{[i \ell,(i+1) \ell) \times[j \ell,(j+1) \ell): i, j \in \mathbb{Z}\} .
$$

For all $i, j \in \mathbb{Z}$, we denote $A_{i j}$ to be the set of links in $A$ whose senders lie in the grid

$$
[i \ell,(i+1) \ell) \times[j \ell,(j+1) \ell) .
$$

We first give a sufficient condition for a set $I$ of links to be independent. Let

$$
K=\left\lceil\sqrt{2}\left((4 \tau)^{-1}\left(\sigma^{-1}-\xi(c \eta)^{-1} R^{\kappa-\beta}\right)\right)^{-1 / \kappa}+\sqrt{2}\right\rceil
$$

Generally, $K$ 's value depends on $R$, we can see that when $\beta=\kappa$,

$$
K=\left\lceil\sqrt{2}\left((4 \tau)^{-1}\left(\sigma^{-1}-\xi(c \eta)^{-1}\right)\right)^{-1 / \kappa}+\sqrt{2}\right\rceil
$$

which is a constant independent of $R$.

Lemma 1. Consider any two nonnegative integers $k_{1}$ and $k_{2}$ which are at most K. Suppose $I$ is a set of links satisfying that for each $i, j \in \mathbb{Z},\left|I \cap A_{i j}\right| \leq 1$ if $i \bmod (K+1)=$ $k_{1}$ and $j \bmod (K+1)=k_{2}$ and $\left|I \cap A_{i j}\right|=0$ otherwise. Then, $I$ is independent. 
Proof. Consider any link $a=(u, v)$. The wanted signal strength is

$$
c\|a\|^{\beta} \cdot \eta\|a\|^{-\kappa}=c \eta\|a\|^{\beta-\kappa} \geq c \eta R^{\beta-\kappa} .
$$

Consider any link $a^{\prime}=\left(u^{\prime}, v^{\prime}\right)$ in $I$ other than $a$. We have $\left\|u^{\prime} u\right\| \geq K \ell$. Therefore,

$$
\left\|u^{\prime} v\right\| \geq\left\|u^{\prime} u\right\|-\|u v\| \geq K \ell-R=(K / \sqrt{2}-1) R .
$$

The total interference to $a$ from all other links in $I$ is at most

$$
\begin{aligned}
& \sum_{(x, y) \in \mathbb{Z}^{2} \backslash\{(0,0)\}} c R^{\beta} \cdot \eta\left(\sqrt{x^{2}+y^{2}} \cdot(K / \sqrt{2}-1) R\right)^{-\kappa} \\
= & c \eta R^{\beta-\kappa}(K / \sqrt{2}-1)^{-\kappa} \sum_{(x, y) \in \mathbb{Z}^{2} \backslash\{(0,0)\}}\left(\sqrt{x^{2}+y^{2}}\right)^{-\kappa} \\
\leq & 4 c \eta R^{\beta-\kappa}(K / \sqrt{2}-1)^{-\kappa}\left(\sum_{i=1}^{\infty} i^{-\kappa}+\sum_{x=1}^{\infty} \sum_{y=1}^{\infty}\left(\sqrt{x^{2}+y^{2}}\right)^{-\kappa}\right) \\
\leq & 4 c \eta R^{\beta-\kappa}(K / \sqrt{2}-1)^{-\kappa}\left(\frac{\kappa\left(1+2^{-\frac{\kappa}{2}}\right)}{\kappa-1}+\frac{\pi 2^{-\kappa / 2}}{2(\kappa-2)}\right) \\
= & 4 \tau c \eta R^{\beta-\kappa}(K / \sqrt{2}-1)^{-\kappa},
\end{aligned}
$$

where

$$
\tau=\frac{\kappa\left(1+2^{-\frac{\kappa}{2}}\right)}{\kappa-1}+\frac{\pi 2^{-\kappa / 2}}{2(\kappa-2)} .
$$

Thus the SINR at the receiver of the link is at least

$$
\frac{c \eta R^{\beta-\kappa}}{\xi+4 \tau c \eta R^{\beta-\kappa}(K / \sqrt{2}-1)^{-\kappa}} \geq \sigma
$$

since

$$
K / \sqrt{2}-1 \geq\left((4 \tau)^{-1}\left(\sigma^{-1}-\xi(c \eta)^{-1} R^{\kappa-\beta}\right)\right)^{-1 / \kappa}
$$

Next, we give a necessary condition for a set $I$ of links to be independent. Let

$$
\omega=\left\lceil\frac{2^{\kappa} P}{\sigma^{2} \xi}+1\right\rceil \text {. }
$$

Lemma 2. For any $I \in \mathcal{I}$ and any $i, j \in \mathbb{Z},\left|I \cap A_{i j}\right| \leq \omega$.

Proof. Let $I_{i j}=I \cap A_{i j}$. Assume $a=(u, v)$ be the shortest link in $I_{i j}$, consider any link $a^{\prime}=\left(u^{\prime}, v^{\prime}\right)$ in $I_{i j}$ other than $a$, the distance between the sender $u^{\prime}$ and $v$ satisfies

$$
\left\|u^{\prime} v\right\| \leq\left\|u^{\prime} u\right\|+\|u v\| \leq\|\sqrt{2} \ell\|+\|u v\| \leq 2 R
$$


The SINR at $a$ from all other links in $I_{i j}$ is at most

$$
\frac{c\|a\|^{\beta} \cdot \eta\|a\|^{-\kappa}}{\sum_{a^{\prime} \in I_{i j} \backslash\{a\}} c\left\|a^{\prime}\right\|^{\beta} \cdot \eta\left\|u^{\prime} v\right\|^{-\kappa}} \leq \frac{\|a\|^{-\kappa}}{\sum_{a^{\prime} \in I_{i j} \backslash\{a\}}\left\|u^{\prime} v\right\|^{-\kappa}} \leq \frac{r^{-\kappa}}{\left(\left|I_{i j}\right|-1\right)(2 R)^{-\kappa}}
$$

Since $\frac{r^{-\kappa}}{\left(\left|I_{i j}\right|-1\right)(2 R)^{-\kappa}} \geq \sigma$, we have $\left|I_{i j}\right| \leq \frac{2^{\kappa}}{\sigma} \frac{R^{\kappa}}{r^{\kappa}}+1<\frac{2^{\kappa}}{\sigma} \frac{P}{\sigma \xi}+1=\frac{2^{\kappa} P}{\sigma^{2} \xi}+1$.

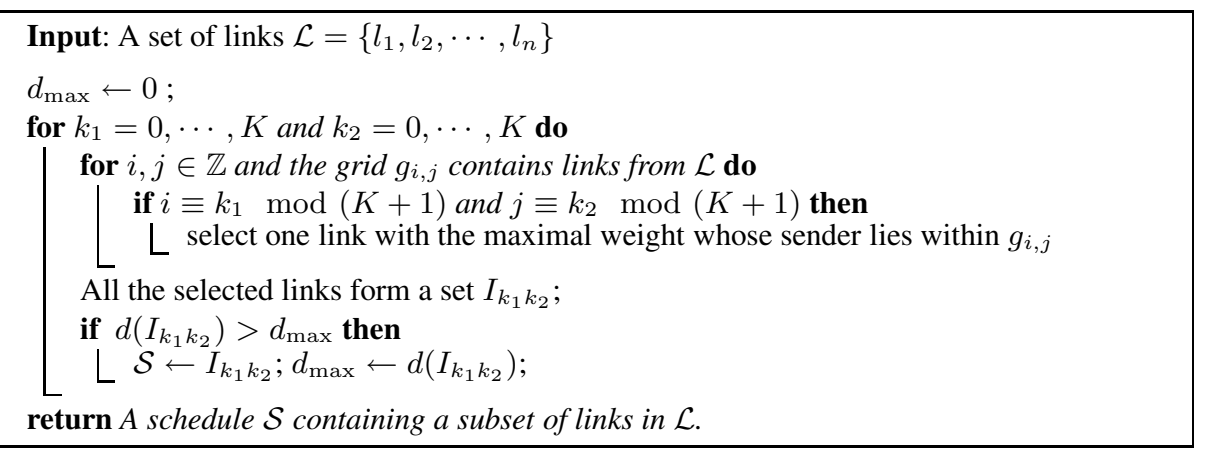

\section{Algorithm 1. Scheduling for MWISL}

Our partition-based scheduling method for input $\mathcal{L}$ is shown in Algorithm 1

The correctness of the algorithm follows from Lemma 1 Next, we derive its approximation bound.

Theorem 1. The approximation ratio of our algorithm for MWISL is at most $(K+$ $1)^{2} \omega$.

Proof. Let $I^{*}$ be a maximum-weighted independent set of links. For any pair of nonnegative integers $k_{1}$ and $k_{2}$, let $I_{k_{1} k_{2}}^{*}$ denote the set of links in $I^{*}$ which lie in the grids

$$
[i \ell,(i+1) \ell) \times[j \ell,(j+1) \ell)
$$

for all $i, j \in \mathbb{Z}$ satisfying that $i \bmod (K+1)=k_{1}$ and $j \bmod (K+1)=k_{2}$. By Lemma2, the set $I_{k_{1} k_{2}}^{*}$ contains at most $\omega$ links from each grid

$$
[i \ell,(i+1) \ell) \times[j \ell,(j+1) \ell)
$$

with $i \bmod (K+1)=k_{1}$ and $j \bmod (K+1)=k_{2}$. Hence,

$$
d\left(I_{k_{1} k_{2}}^{*}\right) \leq \omega d\left(I_{k_{1} k_{2}}\right) \leq \omega \max _{0 \leq k_{1}, k_{2} \leq K} d\left(I_{k_{1} k_{2}}\right) .
$$

Therefore,

$$
d\left(I^{*}\right)=\sum_{0 \leq k_{1}, k_{2} \leq K} d\left(I_{k_{1} k_{2}}^{*}\right) \leq(K+1)^{2} \omega \max _{0 \leq k_{1}, k_{2} \leq K} d\left(I_{k_{1} k_{2}}\right) .
$$

So, the theorem holds. 


\section{Literature Review}

For general graph-based interference model, the maximum throughput link scheduling problem is NP-complete [5]. Both probabilistic scheduling protocols [5, 10, 11] and distributed link scheduling protocols [13, 14] are proposed to maximize the throughput.

For physical interference model, the problem of joint scheduling and power control has been well studied in [3, 4]. In [12], a power-assignment algorithm which schedules a strongly connected set of links in poly-logarithmic time is presented. [6] shows that the scheduling problem without power control under physical interference model, where nodes are arbitrarily distributed is NP-complete. A greedy scheduling algorithm with approximation ratio of $O\left(n^{1-2 /(\Psi(\alpha)+\epsilon)}(\log n)^{2}\right)$, where $\Psi(\alpha)$ is a constant that depends on the path-loss exponent $\alpha$, is proposed in [1]. Notice that this result can only hold when the nodes are distributed uniformly at random in a square of unit area. In [6], an algorithm with a factor $O(g(L))$ approximation guarantee in arbitrary topologies, where $g(L)=\log \vartheta(L)$ is the diversity of the network, is proposed. In [2], an algorithm with approximation guarantee of $O(\log \Delta)$ was proposed, where $\Delta$ is the ratio between the maximum and the minimum distances between nodes. Obviously, it can be arbitrarily larger than $\vartheta(L)$.

Recently, Goussevskaia et al. [7] proposed a method for MWISL in the unweighted case which was claimed to have constant approximation bound. Unfortunately, as observed in Xu and Tang [16], their method [7] works correctly in absence of the background noise. Wan et al. [15] resolved this issue by developing the first correct constantapproximation algorithm. Most Recently, Halldorsson et al. [9] presented a robustness result, showing that constant parameter and model changes will modify the min-length link scheduling result only by a constant. They [8] also studied the scheduling problem under power control.

\section{Conclusions}

We studied MWISL problem under the physical interference model with oblivious power assignment. Some interesting questions are left for future research. The first is to extend our algorithm to deal with a more general path loss model. The second is to develop constant approximation algorithms for MWISL under uniform power and adjustable power assignment settings.

\section{References}

1. Brar, G., Blough, D., Santi, P.: Computationally efficient scheduling with the physical interference model for throughput improvement in wireless mesh networks. In: ACM MobiCom (2006)

2. Chafekar, D., Kumar, V., Marathe, M., Parthasarathy, S., Srinivasan, A.: Approximation Algorithms for Computing Capacity of Wireless Networks with SINR Constraints. In: IEEE INFOCOM, pp. 1166-1174 (2008)

3. Cruz, R., Santhanam, A.: Optimal routing, link scheduling and power control in multihop wireless networks. In: IEEE INFOCOM, vol. 1 (2003) 
4. ElBatt, T., Ephremides, A.: Joint scheduling and power control for wireless ad hoc networks. IEEE Transactions on Wireless Communications 1, 74-85 (2004)

5. Sharma, G., Joo, C., Shroff, N.: Distributed scheduling schemes for throughput guarantees in wireless networks. In: Allerton 2006 (2006)

6. Goussevskaia, O., Oswald, Y., Wattenhofer, R.: Complexity in geometric SINR. In: Proceedings of ACM MobiHoc, pp. 100-109 (2007)

7. Goussevskaia, O., Wattenhofer, R., Halldórsson, M.M., Welzl, E.: Capacity of Arbitrary Wireless Networks. In: IEEE INFOCOM 2009 (2009)

8. Halldórsson, M.: Wireless scheduling with power control. In: Fiat, A., Sanders, P. (eds.) ESA 2009. LNCS, vol. 5757, pp. 361-372. Springer, Heidelberg (2009)

9. Halldorsson, M., Wattenhofer, R.: Wireless Communication is in APX. In: Automata, Languages and Programming, pp. 525-536

10. Joo, C., Shroff, N.: Performance of random access scheduling schemes in multi-hop wireless networks. In: IEEE INFOCOM (2007)

11. Lin, X., Rasool, S.: Constant-time distributed scheduling policies for ad hoc wireless networks. In: IEEE CDC (2006)

12. Moscibroda, T., Wattenhofer, R.: The Complexity of Connectivity in Wireless Networks. In: IEEE INFOCOM (2006)

13. Penttinen, A., Koutsopoulos, I., Tassiulas, L.: Low-complexity distributed fair scheduling for wireless multi-hop networks. In: WiOPT 2005: First Workshop on Resource Allocation in Wireless Networks, RAWNET (2005)

14. Sanghavi, S., Bui, L., Srikant, R.: Distributed link scheduling with constant overhead. In: ACM SIGMETRICS, pp. 313-324. ACM Press, New York (2007)

15. Wan, P., Jia, X., Yao, F.: Maximum Independent Set of Links under Physical Interference Model. In: Liu, B., Bestavros, A., Du, D.-Z., Wang, J. (eds.) WASA 2009. LNCS, vol. 5682, pp. 169-178. Springer, Heidelberg (2009)

16. Xu, X., Tang, S.: A constant approximation algorithm for link scheduling in arbitrary networks under physical interference model. In: Proceedings of the 2nd ACM International Workshop on Foundations of Wireless Ad Hoc and Sensor Networking and Computing, pp. 13-20. ACM, New York (2009) 